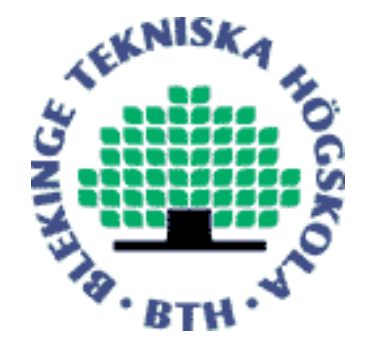

Copyright (C) 2014 IEEE.

Citation for the published paper:

RFID - Hybrid Scene Analysis-Neural Network System for 3D Indoor Positioning optimal system arrangement approach

Bartosz Jachimczyk, Damian Dziak, Wlodek J. Kulesza

Instrumentation and Measurement Technology Conference (I2MTC) Proceedings, 2014 IEEE International

2014 Montevideo

This material is posted here with permission of the IEEE. Such permission of the IEEE does not in any way imply IEEE endorsement of any of BTH's products or services Internal or personal use of this material is permitted. However, permission to reprint/republish this material for advertising or promotional purposes or for creating new collective works for resale or redistribution must be obtained from the IEEE by sending a blank email message to pubs-permissions@iee.org.

By choosing to view this document, you agree to all provisions of the copyright laws protecting it. 


\title{
RFID - Hybrid Scene Analysis-Neural Network System for 3D Indoor Positioning
}

\author{
Optimal System Arrangement Approach
}

\author{
Bartosz Jachimczyk, Damian Dziak \\ Gdansk University of Technology \\ Faculty of Electrical and Control Engineering \\ Gdansk, Poland \\ bjachimczyk@ely.pg.gda.pl,ddziak@ely.pg.gda.pl
}

\author{
Wlodek J. Kulesza \\ Blekinge Institute of Technology \\ Institute of Applied Signal Processing \\ Karlskrona, Sweden \\ wlodek.kulesza@bth.se
}

\begin{abstract}
The purpose of this research is to find an optimal number and configuration of readers in RFID based 3D Indoor Positioning System. The system applies a Hybrid Scene Analysis Neural Network algorithm to estimate target's position with a desired accuracy. The system's accuracy and cost depend on a number of utilized readers and their arrangement. Readers' deployment is crucial for the localization accuracy too. The system optimization enhances the system cost-efficiency. The arrangement analysis was based on simulations and validated by physical experiment. The results of this research define a tradeoff between a number of readers and their deployment and the system performance in terms of localization accuracy.
\end{abstract}

Keywords-optimization; radiofrequency identification; reader configuration; RFID network planning;

\section{INTRODUCTION}

An effective localization and tracking of an object or person in a 3D indoor space can be carried out using a short range wireless technology called Radio Frequency IDentification, RFID. Systems based on this technology are widely used in healthcare facilities [1][2], hospitals [3] and kindergartens [4]. Such systems can be used for emergency monitoring of tumble detections of solitary people [5]. RFID systems are also used for tracking and recognition of products and goods in warehouses [6][7] or markets [8]. Localization accuracy is a crucial issue in all these applications.

Many algorithms and techniques are applied in RFID positioning systems. One of them is Scene Analysis, SA, technique, which is a type of RFID fingerprinting technique [9][10]. It is based on comparison of actual measurements of the tag's Received Signal Strength, RSS, or Time of Arrival, ToA, with a pattern called a radio map. This map is created from a set of measurements performed beforehand, with tags placed at points of given coordinates. To improve 3D indoor target localization, SA technique can be combined with Neural Network, NN [11]. The analysis of this solution considered a case of eight readers located in specified coordinates of an indoor space. The estimated system accuracy of about $26 \mathrm{~cm}$ proved advantages and usefulness of the solution for many applications.
This research is solving a Readers Planning Optimization, RPO, problem by searching for an optimal configuration of the RFID Scene Analysis - Neural Network, SA-NN, 3D indoor positioning system. To be cost-efficient, the system should assure a desired 3D localization quality for a minimal number of readers. The results of simulation and physical experiment analysis show how the localization accuracy depends not only on the number of readers, but also on their deployment.

\section{SURVEY OF RELATED WORKS}

Sensor deployment optimization methods can refer to different localization and tracking systems using a variety of sensor types. Chen et al. propose a method for planning the position of multiple stereo sensors in an indoor space [12]. The solution, based on a combination of a greedy algorithm and a linear programing model, minimizes the number of vision sensors needed to cover the target object in an indoor environment.

In the cases of acoustic systems, the optimal placement of sensors can be found by means of the probabilistic analysis [13]. The proposed algorithm optimally displaces the sensor to maximize detection probability.

There have been researchers who aimed to determine how localization quality depends on the RFID readers' configuration. In [14] authors propose an optimal reader configuration for system localization performance by dividing an indoor space into several independent zones characterized by a sensing rate parameter.

Artificial intelligence methods and different algorithms are commonly used for the optimal reader deployment in a 3D indoor space. In [1][15][16] the main research objective was to develop a maximal covering optimization method for placement of a limited number of RFID readers. The approach based on Genetic Algorithms, determines the optimal placement of readers on the whole healthcare facility floor [1]. The Particle Swarm Optimization, PSO, methods are also suitable for RPO problems due to their high efficiency, fast convergence and strong robustness. In [15] Gong et al. used the PSO methods with a tentative reader elimination operator, to solve a RPO problem. In [16] multi-swarm PSO, which is an 
extended version of a typical PSO solver, was presented for a 2D RFID networking planning problem.

\section{Problem Statement AND The Proposed Solution METHOD}

The results of the previous work [11] show that the RFID 3D indoor localization system, based on multiple readers and a hybrid SA-NN algorithm is able to estimate a target's position with a desired accuracy. However, the system cost-efficiency depends on its configuration, i.e. a number of readers, antennas, wiring etc. Therefore, it has become crucial to define how the system performance depends on its configuration. Moreover, since the readers' arrangement affects the system performance, further analysis is required to find a tradeoff between the performance quality and costs.

The cost of the system can be minimized by reducing the number of required readers. The analysis of all possible arrangements for a different number of readers can clearly determine their optimal deployment. It provides a minimal number of readers for a desired localization accuracy.

In the case of RFID based 3D Indoor Positioning System, the SA-NN algorithm compares actual RSS or ToA measurements with RSS/ToA patterns, also called RSS/ToA map. The applied NN structure consists of three layers. The first one, an input layer, includes a number of inputs corresponding to active readers. The size of the second hidden layer is set to 23 neurons. The third output layer of network includes three neurons corresponding to the estimated tag's 3D positioning coordinates. Activation functions used in the hidden layer are hyperbolic tangent sigmoid functions. Moreover, the linear function is used in the output layer. The Neural Network training process is based on the back propagation function, which updates weight and bias values according to the gradient descent momentum and adaptive learning rate. MSE is used as the best performance function [11]. The SA-NN model using RSS was validated by the comparison of the simulation results with the results obtained from the measurements. Eight RFID readers located in all corners of the cuboidal area of dimensions $5.13 \mathrm{~m} \times 4.5 \mathrm{~m} \times 2 \mathrm{~m}$ were used in the experiments. The virtual results matched the physical experiment results [11].

In this research the analysis performed using the same system in the same area $5.13 \mathrm{~m} \times 4.5 \mathrm{~m} \times 2 \mathrm{~m}$ as in [11]. The room model with possible readers' deployment in the room corners is presented in Fig. 1. The six test cases correspond to three, four, five, six, seven and eight active readers respectively. Numbers of possible different deployment configurations for each test case are shown in Table I. Due to geometrical features of the room and radio wave propagation properties, the configurations can be grouped into a number of equivalent scenarios. For example, in the test case with four active readers, the configurations (A B A' B') and (C D C' D') are classified as a single scenario.

In this paper we apply a heuristic method based on an investigation of virtual and real systems to find out which cases and which scenarios are the most suitable for a desired localization accuracy and cost. To examine how system configuration affects the localization accuracy, simulations

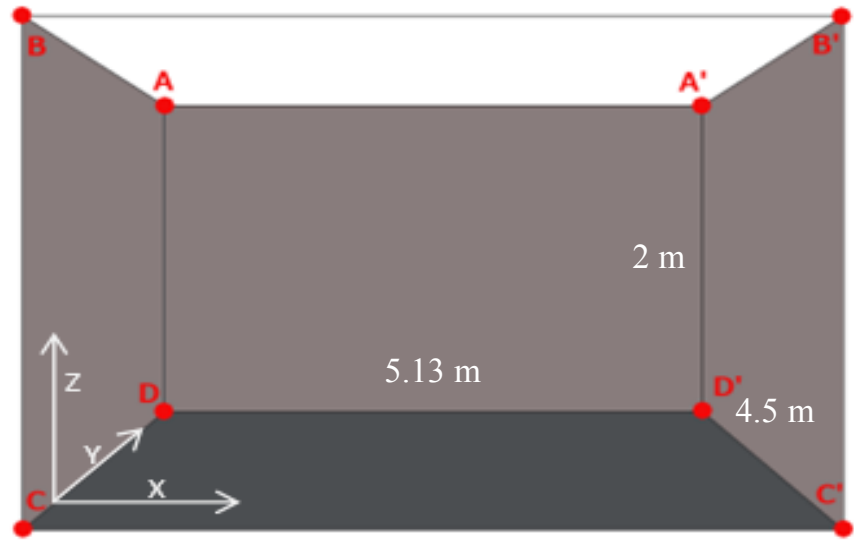

Fig. 1. Room model with readers' deployment

using Matlab are performed. Furthermore, a real system is examined. The results of simulations and measurement are compared to confirm the findings. The method covers three main approaches. The first approach deals with performance analysis of test cases. The following approach evaluates performances of possible scenarios. And the last approach searches for a cost-effective solution.

The average localization accuracy in each configuration is defined by coordinate mean values of estimation uncertainty calculated as:

$$
\begin{gathered}
\bar{d}_{x}=\frac{1}{J \cdot I} \sum_{j=1}^{J} \sum_{i=1}^{I}\left(x_{j, i}-\hat{x}_{j, i}\right), \\
\bar{d}_{y}=\frac{1}{J \cdot I} \sum_{j=1}^{J} \sum_{i=1}^{I}\left(y_{j, i}-\hat{y}_{j, i}\right), \\
\bar{d}_{z}=\frac{1}{J \cdot I} \sum_{j=1}^{J} \sum_{i=1}^{I}\left(z_{j, i}-\hat{z}_{j, i}\right),
\end{gathered}
$$

where $J$ is the number of test sets, $I$ is the number of tested tags coordinates, $x_{i j}, y_{i j}$, and $z_{i j}$, are coordinates of each $i j$ tag position, $\hat{x}, \hat{y}, \hat{z}$ refer to estimated tag's coordinates, $\bar{d}_{x}, \bar{d}_{y}, \bar{d}_{z}$, represent uncertainty mean values in $x, y, z$ axes respectively.

TABLE I. NUMBERS OF CONFIGURATIONS AND SCENARIOS OF READERS' DEPLOYMENT FOR EACH TEST CASE

\begin{tabular}{|c|c|c|}
\hline \multirow{2}{*}{$\begin{array}{c}\text { Test case - } \\
\text { Number of active readers }\end{array}$} & \multicolumn{2}{|c|}{ Number of } \\
\cline { 2 - 3 } configuration & scenarios \\
\hline 3 & 56 & 7 \\
\hline 4 & 70 & 14 \\
\hline 5 & 56 & 7 \\
\hline 6 & 28 & 7 \\
\hline 7 & 8 & 1 \\
\hline 8 & 1 & 1 \\
\hline
\end{tabular}


To evaluate the performance for each scenario, mean value $\bar{d}$ and respective standard deviation were calculated as follows:

$$
\bar{d}=\sqrt{\bar{d}_{x}^{2}+\bar{d}_{y}^{2}+\bar{d}_{z}^{2}}
$$

To evaluate localization quality by means of simulation, for each test case and for each reader configuration, 100 different sets containing 100 uniformly and uniquely distributed tags' coordinates were simulated.

The experiment on a real system was carried out in a cuboidal room of the same dimensions as the virtual room equipped with basic office furniture. To evaluate localization quality, the experiment was performed for 20 randomly selected positions of the target tag. To reduce interferences during the real experiment only one person was present in the room.

\section{APPROACH 1 - ANALYSIS BASED ON NUMBER OF ACTIVE READER - TEST CASES}

In this approach we investigate performance of each test case under the conditions described in the previous section. The simulation and measurement results are used to find the cases with best localization quality.

\section{A. Simulation Results}

Simulation results presented in Fig. 2 show mean uncertainty and standard deviation of the estimated tags' localization. The results indicate a significant correlation between a number of active readers and estimated localization accuracy. The results show that for the SA-NN system using seven active RFID readers, the average localization uncertainty is $0.5 \mathrm{~cm}$ with standard deviation of $50.0 \mathrm{~cm}$, when the next best accuracy for eight readers is characterized by uncertainty mean value of $7.0 \mathrm{~cm}$ and standard deviation is equal to $50.0 \mathrm{~cm}$.

Simulation results of test cases from three up to seven active readers follow the expectation that with a higher number of active readers, localization accuracy would be better. However, the results for the test case with eight active readers

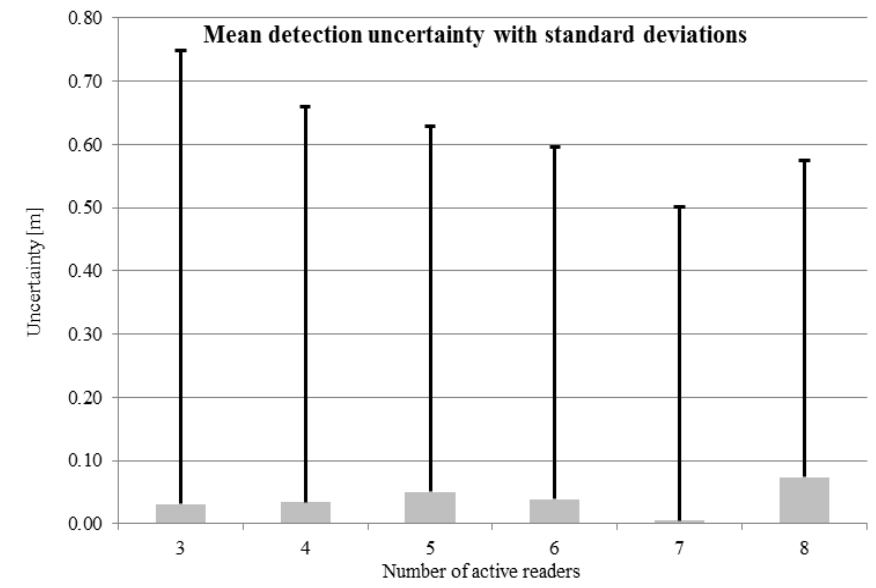

Fig. 2. Simulation results of mean localization uncertainty of test cases with different numbers of active readers show slightly less accuracy than the results for the test case with seven active readers.

\section{B. Experimental Results}

In this approach average localization uncertainty and its standard deviation are measured for the three test cases: fourth, sixth and eighth. Table II shows the results of the best scenarios of test cases six and eight. To show differences in the fourth test case the table presents results of scenario 1, 11 and 12 with best, respectively medium and worst performances.

The experimental results show a correlation between a number of active readers and estimated localization accuracy. The performance results of the SA-NN system using eight active readers achieved mean localization uncertainty of $24.2 \mathrm{~cm}$ with a standard deviation of $48.9 \mathrm{~cm}$. The next best performance for the best scenario when four active readers is characterized by an uncertainty of $22.0 \mathrm{~cm}$ and a standard deviation of $54.3 \mathrm{~cm}$. The data in Table II indicates that for the test case with eight active readers, localization accuracy is slightly better than the test case with four active readers. The presented results also show that in the case of the best scenario with six active readers, the mean localization uncertainty is $27.2 \mathrm{~cm}$ with standard deviation of $55.4 \mathrm{~cm}$, which is worse than for the best scenario for four active readers.

\section{Discussion}

The results presented in the preceding sections confirm a correlation between a number of active readers and estimated localization accuracy. Fig. 3 presents a relationship between mean localization uncertainty and a number of used RFID readers for the best scenarios of the three chosen test cases: eight, six and four active readers. Experiment and simulation results for four and eight active readers differ slightly. However, in the case of eight readers, the experimental results show better localization uncertainty than for four readers, but the difference is less than $4.5 \%$.

TABLE II. EXPERIMENTAL RESULTS OF LOCALIZATION UNCERTAINTY FOR FIVE SCENARIOS

\begin{tabular}{|c|c|c|c|c|c|}
\hline \multirow{2}{*}{$\begin{array}{c}\text { Number } \\
\text { of } \\
\text { active } \\
\text { readers }\end{array}$} & \multirow{2}{*}{ } & \multicolumn{2}{|c|}{ Uncertainty } & \multirow{2}{*}{$\begin{array}{c}\text { Configuration } \\
\text { sample }\end{array}$} & \multirow{2}{*}{ 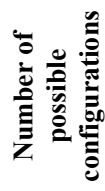 } \\
\hline & & $\begin{array}{c}\text { Mean } \\
{[\mathrm{m}]}\end{array}$ & $\begin{array}{r}S T D \\
{[\mathrm{~m}]}\end{array}$ & & \\
\hline \multirow{3}{*}{4} & 1 & 0.22 & 0.54 & A B A' B' & 2 \\
\hline & 11 & 0.25 & 0.59 & A B C D' & 8 \\
\hline & 12 & 0.30 & 0.64 & A C B' D' & 2 \\
\hline 6 & 1 & 0.27 & 0.55 & A B C A' B' C' & 4 \\
\hline 8 & 1 & 0.24 & 0.49 & A B C D A' B' C' D' & 1 \\
\hline
\end{tabular}




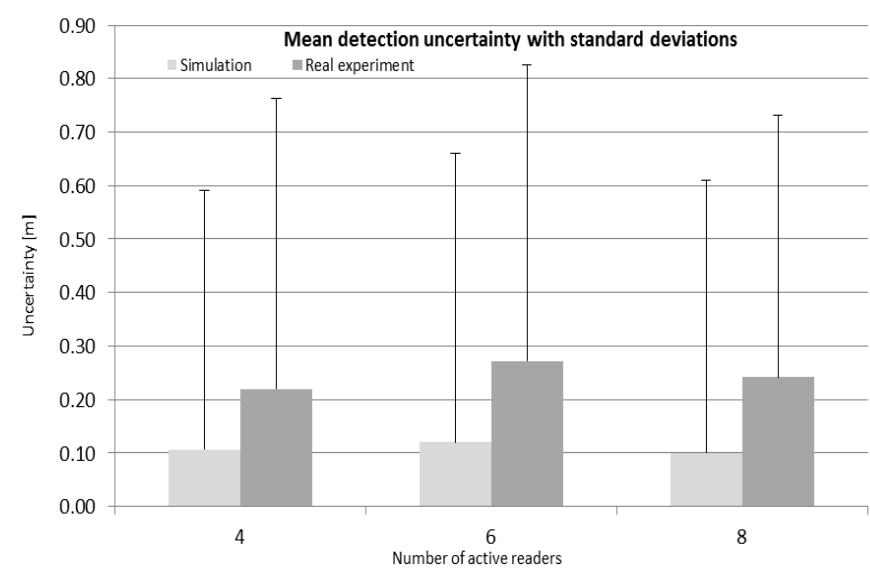

Fig. 3. Simulation and experiment results of mean localization uncertainty for three best test cases

\section{APPROACH 2 -ANALYSIS OF DIFFERENT SCENARIOS}

In this approach we investigate the performance of different scenarios. The issue is to compare performances of different scenarios within one test case. But also comparison of the best performing scenarios of different test cases is an issue. The simulation and measurement results are used to find the scenario with the best localization quality.

\section{A. Simulation Results}

Table III presents mean localization uncertainty and its standard deviation, for each possible scenario when four RFID readers are active. These results show significant differences in localization accuracy between individual scenarios.

TABLE III. SIMULATION RESULTS OF LOCALIZATION UNCERTAINTY FOR ALL SCENARIOS OF THE TEST CASE WITH FOUR ACTIVE READERS

\begin{tabular}{|c|c|c|c|c|}
\hline \multirow{2}{*}{ Scenario } & \multicolumn{2}{|c|}{ Uncertainty } & \multirow{2}{*}{$\begin{array}{c}\text { Configuration } \\
\text { sample }\end{array}$} & \multirow{2}{*}{ 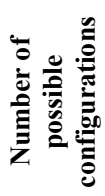 } \\
\hline & $\begin{array}{c}\text { Mean } \\
{[\mathrm{m}]}\end{array}$ & $\begin{array}{r}S T D \\
{[\mathrm{~m}]}\end{array}$ & & \\
\hline 1 & 0.11 & 0.49 & $\mathrm{~A} \mathrm{~B} \mathrm{~A}^{\prime} \mathrm{B}^{\prime}$ & 2 \\
\hline 2 & 0.15 & 0.63 & B C C' D' & 8 \\
\hline 3 & 0.16 & 0.62 & $\mathrm{C} \mathrm{B}^{\prime} \mathrm{C}^{\prime} \mathrm{D}^{\prime}$ & 8 \\
\hline 4 & 0.16 & 0.64 & $\mathrm{C} \mathrm{A}^{\prime} \mathrm{C}^{\prime} \mathrm{D}^{\prime}$ & 8 \\
\hline 5 & 0.17 & 0.58 & $A C^{\prime} D^{\prime}$ & 8 \\
\hline 6 & 0.18 & 0.71 & $\mathrm{~B}^{\mathrm{C}} \mathrm{B}^{\prime} \mathrm{C}^{\prime}$ & 2 \\
\hline 7 & 0.16 & 0.61 & $\mathrm{~B} \mathrm{C} \mathrm{A}^{\prime} \mathrm{C}^{\prime}$ & 8 \\
\hline 8 & 0.17 & 0.62 & A B C C' & 8 \\
\hline 9 & 0.12 & 0.54 & $\mathrm{~A} \mathrm{C} \mathrm{A}^{\prime} \mathrm{C}^{\prime}$ & 2 \\
\hline 10 & 0.20 & 0.60 & B C A' D' & 2 \\
\hline 11 & 0.16 & 0.59 & A B C D' & 8 \\
\hline 12 & 0.26 & 0.62 & A C B' D' & 2 \\
\hline 13 & 0.19 & 0.88 & A B C D & 2 \\
\hline 14 & 0.15 & 0.60 & C D A' B' & 2 \\
\hline
\end{tabular}

These differences are also observed between best scenarios of different test cases. Some best scenarios with fewer readers can show better localization accuracy then scenarios with more readers. For instance from Fig. 4 and Fig. 5 we can see that the best scenario in the case of four active readers is better than the best scenario when five RFID readers are present.

Table IV shows mean localization uncertainty and its standard deviation for the best scenario in each test case. The lowest mean uncertainty is obtained for the case when all eight readers are active. However, considering the standard deviation, the best results are obtained for the scenario with four active readers. The mean values of localization uncertainties for four and eight active readers are: $11.0 \mathrm{~cm}$ and $10.0 \mathrm{~cm}$ respectively with standard deviation of $49.0 \mathrm{~cm}$ and $51.0 \mathrm{~cm}$ respectively. Furthermore, the analysis shows that differences in mean uncertainty among the best scenarios of test cases with eight, seven, and four active readers do not exceed $3 \%$.

\section{B. Experimental Results}

In this approach we investigate the average localization accuracy of three scenarios when four RFID readers are active. To discover variations of the three scenarios: 1,11 and 12 are tested. Results presented in Table II show mean localization uncertainty and its standard deviation for the examined scenarios.

The best mean localization uncertainty is achieved for the scenario one when all four readers are mounted either on the ceiling (A B A' B') or near the floor (C D C' D'). In this scenario the real mean localization uncertainty is $22.0 \mathrm{~cm}$ with standard deviation of $54.3 \mathrm{~cm}$.

The second examined scenario 11 with a sample deployment (D A' B' C'), when three tags are placed in the corners of the shorter wall and fourth tag is on the opposite wall, is characterized by mean localization uncertainty of $24.6 \mathrm{~cm}$ and standard deviation of $58.7 \mathrm{~cm}$.

The last tested scenario 12 with a sample deployment (B D A' C'), when tags are placed diagonally of the examined room, mean localization uncertainty was $22.9 \mathrm{~cm}$ with $64.1 \mathrm{~cm}$ standard deviation. Those results are consistent with the simulation results.

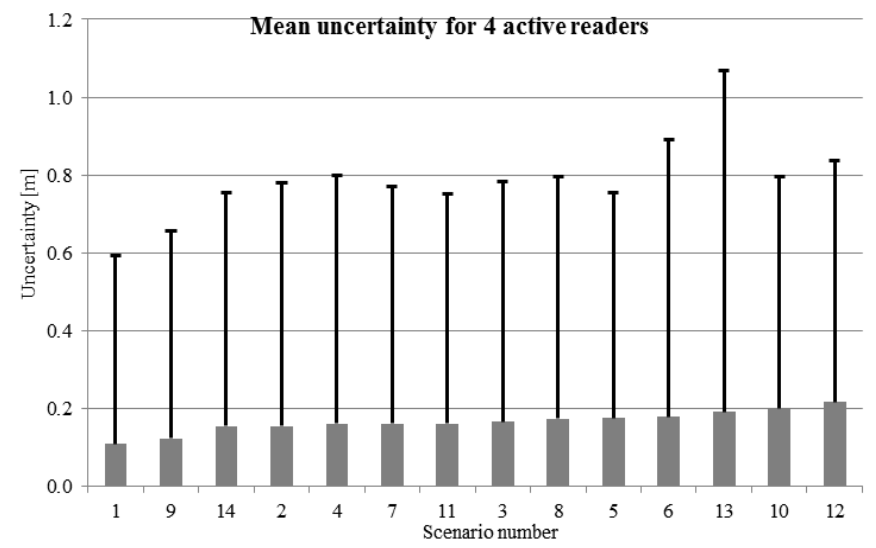

Fig. 4. Simulation results of mean uncertainty for all scenarios of test case with four active readers: in order from lowest to biggest mean uncertainty 


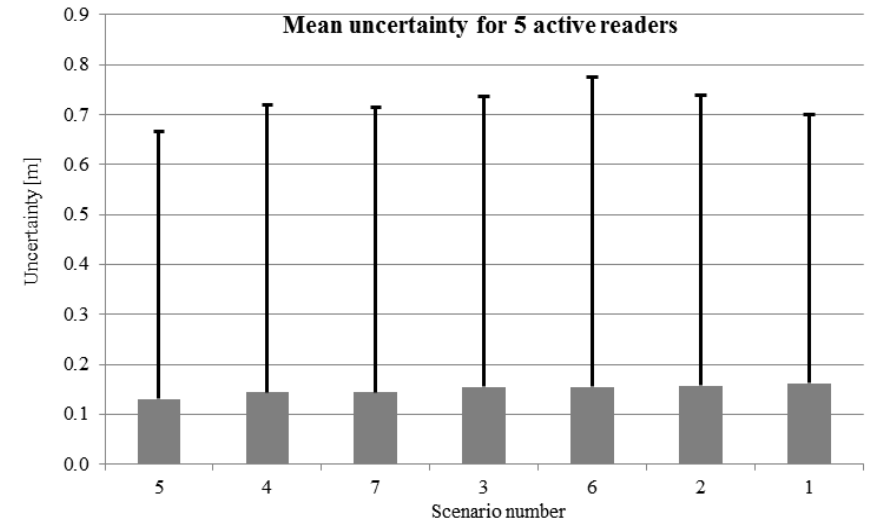

Fig. 5. Simulation results of mean uncertainty for all scenarios of the test case with five active readers; in order from lowest to biggest mean uncertainty

\section{Discussion}

The results presented in preceding sections confirm that there is also a strong correlation between the arrangement of used RFID readers and localization accuracy. Results from Fig. 6 confirm that the experimental results follow the relationship among different scenarios' performances from the simulations. There is a clear correlation between virtual and physical experiments' results for four active RFID readers. The best, medium, and worst localization accuracies of examined scenarios in simulation and physical experiment correspond to each other.

\section{APPROACH 3 - COST-EFFECTIVNESS ANALYSIS}

The RPO problem refers not only to system complexity and localization accuracy, but also to the system cost. The aim of the cost-effective solution is to localize the target in a $3 \mathrm{D}$ indoor space with maximum accuracy using a minimal number of readers. Therefore, additional analysis is needed to define a tradeoff between the cost of the system and its performance quality [1][15].

TABLE IV. SIMULATION RESULT OF LOCALISATION UNCERTAINTY FOR THE BEST SCENARIO OF EACH SIMULATION TEST CASE

\begin{tabular}{|c|c|c|c|c|c|}
\hline \multirow{2}{*}{$\begin{array}{c}\text { Number } \\
\text { of } \\
\text { active } \\
\text { readers }\end{array}$} & \multirow{2}{*}{ 悉 } & \multicolumn{2}{|c|}{ Uncertainty } & \multirow{2}{*}{$\begin{array}{c}\text { Configuration } \\
\text { sample }\end{array}$} & \multirow{2}{*}{ 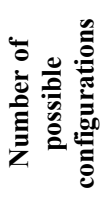 } \\
\hline & & $\begin{array}{c}\text { Mean } \\
{[\mathrm{m}]}\end{array}$ & $\begin{array}{r}S T D \\
{[\mathrm{~m}]}\end{array}$ & & \\
\hline 3 & 5 & 0.15 & 0.66 & C D B' & 8 \\
\hline 4 & 1 & 0.11 & 0.49 & A B A' B' & 2 \\
\hline 5 & 5 & 0.13 & 0.54 & A B $A^{\prime} B^{\prime} C^{\prime}$ & 8 \\
\hline 6 & 1 & 0.12 & 0.54 & A B C A' B' $C^{\prime}$ & 4 \\
\hline 7 & 1 & 0.12 & 0.49 & A B D A $A^{\prime} B^{\prime} C^{\prime} D^{\prime}$ & 8 \\
\hline 8 & 1 & 0.10 & 0.51 & A B C D A' B' $C^{\prime} D^{\prime}$ & 1 \\
\hline
\end{tabular}

The simulation results shown in Section $\mathrm{V}$ show that the test case when four RFID readers in their optimized scenario assures better accuracy compared to a more expensive eight active reader solution. Considering the three best scenarios for test cases with four, eight, and seven active readers, localization accuracy differs only by about $3 \%$. However, the system cost for eight and four active readers varies significantly, and differences can reach $40 \%$ in favor of four readers. Then, substantial savings, without compromising the system localization accuracy, can be reached by applying four readers arranged in (A B A' B') or (C D C' D').

The experimental results presented in Section $\mathrm{V}$ show that using SA-NN algorithm, the best mean localization uncertainty, is achieved for the case when all four RFID readers are placed either near the ceiling or floor corners. However, considering the standard deviation, the best results are observed for the test case with eight active readers. The localization accuracy with four and eight active readers differs only by about $4 \%$, but the cost concerning a system with four active readers can be $40 \%$ smaller than a system with eight readers. Then, we can conclude that the system with four readers placed either near the ceiling ( $\left.\mathrm{A} B \mathrm{~A}^{\prime} \mathrm{B}^{\prime}\right)$ or near the floor (C D C' D') is the most suitable solution.

The presented analysis leads to the conclusion that both simulation and experimental results prove that the system consisting of four active readers, mounted near the ceiling (A B A' B'), or near the floor (C D C' D'), provides the optimal solution to a given problem.

\section{CONCLUSION}

The aim of the paper was to discover the most suitable arrangement of the RFID based 3D Indoor Positioning System. For this purpose we applied a heuristic method using the SA$\mathrm{NN}$ model tested under various conditions. The result of this research defines an optimal number of RFID readers and their arrangement for a cost-effective solution.

To achieve the research goal three approaches were investigated. In Approach 1 we investigated performance of all test cases corresponding to a different number of readers under

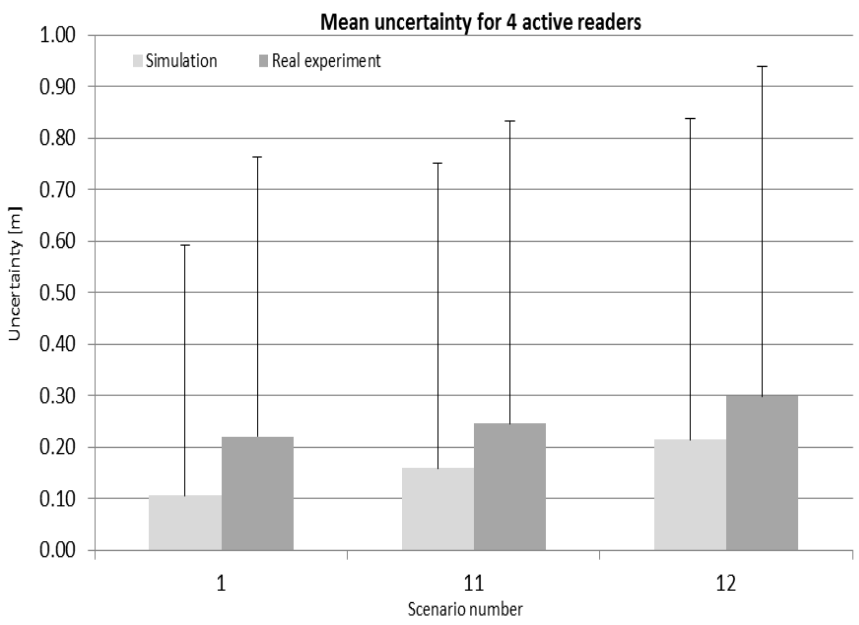

Fig. 6. Simulation and experimental results of mean uncertainty for three scenario of test case with four active readers 
the desired conditions. In Approach 2 we examined the performance of different scenarios within various test cases. The aim of Approach 3 was to find the cost-effective solution assuring best accuracy using a minimal number of readers.

All three approaches were based on the analysis of performances of virtual and real RFID based 3D Indoor Positioning Systems. The simulations and measurements were performed in the same area of size $5.13 \mathrm{~m} \times 4.5 \mathrm{~m} \times 2 \mathrm{~m}$. The six test cases correspond to the various numbers of active readers implemented, from three to eight. Due to geometrical features of the room and radio wave propagation properties, readers' placements in each test case were grouped into a number of equivalent scenarios.

The simulation results indicate a significant correlation between a number of active readers and localization performances in the cases with three to seven active readers. While the localization accuracy for the SA-NN positioning system using seven active RFID readers was better, even when compared to the configuration using eight readers. The average localization uncertainty for seven readers was $0.5 \mathrm{~cm}$ with a standard deviation of $50.0 \mathrm{~cm}$, whereas, for the eight reader solution, the respective results were $7.0 \mathrm{~cm}$ and $50.0 \mathrm{~cm}$. However, good localization accuracy was obtained while optimally placing four readers, resulting with an uncertainty of $11.0 \mathrm{~cm}$ mean value and $49.0 \mathrm{~cm}$ standard deviation. The experimental results verified a significant correlation between a number of active readers and localization performance. The best localization accuracy is for eight active readers; however the localization accuracy for four active readers was only lower by $4.5 \%$.

Our research on the performance of the SA-NN localization system shows crucial meaning of readers' arrangement. For instance, the smallest localization uncertainty including mean value and standard deviation was obtained while using four readers arranged in the way that readers are placed in (A B A' $\left.\mathrm{B}^{\prime}\right)$ or $\left(\mathrm{C} \mathrm{D} \mathrm{C}^{\prime} \mathrm{D}^{\prime}\right)$ position, with a mean value of $11.0 \mathrm{~cm}$ and $49.0 \mathrm{~cm}$ standard deviation. The experimental results verified this however with worse uncertainty of $22.0 \mathrm{~cm}$ with a standard deviation of $54.3 \mathrm{~cm}$.

Research results show that the difference in localization accuracy among the best scenarios, when eight, seven or four readers are active, does not exceed $3 \%$ in case of simulation. For the experimental results the difference between the two best scenarios with four and eight active readers, do not exceed $4.5 \%$. However costs associated with the equipment can by $40 \%$ lower in case of four active readers than of eight readers. This leads to the conclusion that the solution with four active readers located in ceiling corners is the most cost-effective.

The additional advantage of the arrangement of readers on the ceiling (A B A' B') is its user-friendliness. Moreover, all readers located in this way are more robust and avoid obstacles that would most likely occur if positioned on the floor.

Further research may concern the development of SA-NN technique applying a Real Time Location System, enabling ToA measurements. Complementary research may concern the application of another artificial intelligence method with SA technique, i.e. swarm intelligence or fuzzy rules, and compare it with SA-NN results.

\section{REFERENCES}

[1] A. Oztekin, F. M. Pajouh, D. Delen, and L. K. Swim, "An RFID network design methodology for asset tracking in healthcare," Decision Support Systems, vol. 49, no. 1, pp. 100-109, Apr. 2010.

[2] J. Chen, I. D. Olayanju, O. P. Ojelabi, and W. Kulesza, "RFID Multitarget Tracking Using the Probability Hypothesis Density Algorithm for a Health Care Application," in in IT Revolutions, Berlin Heidelberg, 2012, pp. 95-105.

[3] A. Pietrabissa, C. Poli, D. G. Ferriero, and M. Grigioni, "Optimal planning of sensor networks for asset tracking in hospital environments," Decision Support Systems, vol. 55, no. 1, pp. 304-313, Apr. 2013.

[4] A. R. Al-Ali, F. A. Aloul, N. R. Aji, A. A. Al-Zarouni, and N. H. Fakhro, "Mobile RFID Tracking System," in 3rd International Conference on Information and Communication Technologies: From Theory to Applications, 2008. ICTTA 2008, 2008, pp. 1-4.

[5] Quanyi Ge, Yi Chai, ,RFID Emergency System for Tumble Detection of Solitary People," Bachelor Thesis, Dept. Elect. Eng, Blekinge Institute of Technology, Karlskrona, Sweden, 2012. The thesis is availible in: http://www.bth.se/fou/cuppsats.nsf/all/8e4f951787c600afc1257a350045 8e1e/\$file/bth2012chai.pdf [Oct. 15, 2013].

[6] W. W. Y. Ng, L. Lin, P. P. K. Chan, and D. S. Yeung, "3D Goods allocation in warehouse with L-GEM based 3-D RFID positioning," in 2011 International Conference on Machine Learning and Cybernetics (ICMLC), 2011, vol. 1, pp. 324-329.

[7] L. Minbo, G. Shengxi, C. Guangyu, and Z. Zhu, "A RFID-based Intelligent Warehouse Management System Design and Implementation," in 2011 IEEE 8th International Conference on eBusiness Engineering (ICEBE), 2011, pp. 178-184.

[8] D. Wang and W. H. Ip, "Review on modeling and optimization problems about RFID technology and applications," in Control and Decision Conference (CCDC), 2013 25th Chinese, 2013, pp. 12581263.

[9] T. Jingwangsa, S. Soonjun, P. Cherntanomwong, "Comparison between innovative approaches of RFID based localization using fingerprinting techniques for outdoor and indoor environments," in 2010 The 12th ICACT, 2010, vol. 2, pp. 1511-1515.

[10] A. Papapostolou and H. Chaouchi, "WIFE: Wireless Indoor Positioning Based on Fingerprint Evaluation," in NETWORKING 2009, Berlin Heidelberg, 2009, pp. 234-247.

[11] B. Jachimczyk, D. Dziak, W. Kulesza, "Performance analysis of an RFID-based 3D indoor positioning system combining scene analysis and neural network methods,"in The Scientific Papers of Faculty of Electrical and Control Engineering Gdansk University of Technology, vol. 34, pp. 29-33, Sept. 2013.

[12] J. Chen, S. Khatibi, and W. Kulesza, "Planning of a Multi Stereo Visual Sensor System - Depth Accuracy and Variable Baseline Approach," in 3DTV Conference, 2007, 2007, pp. 1-4.

[13] R. Stolkin and I. Florescu, "Probabilistic analysis of a passive acoustic diver detection system for optimal sensor placement and extensions to localization and tracking," in OCEANS 2007, 2007, pp. 1-6.

[14] S. Jeon and J. Park, "A RFID Reader Configuration with an Enhanced Recognition Property for Indoor Positioning," in Fifth International Joint Conference on INC, IMS and IDC, 2009. NCM '09, 2009, pp. 166-169.

[15] Y.-J. Gong, M. Shen, J. Zhang, O. Kaynak, W.-N. Chen, and Z.-H. Zhan, "Optimizing RFID Network Planning by Using a Particle Swarm Optimization Algorithm With Redundant Reader Elimination," IEEE Transactions on Industrial Informatics, vol. 8, no. 4, pp. 900-912, 2012.

[16] H. Chen, Y. Zhu, and K. Hu, "RFID networks planning using a multiswarm optimizer," in Control and Decision Conference, 2009. CCDC '09. Chinese, 2009, pp. 3548-3552. 